\title{
Achieving Cost Minimization and Fairness in Multi-Supplier Smart Grid Environment
}

\author{
Amna Malik ${ }^{1}$, Zain Ali ${ }^{2}$, Ahmed Bilal Awan ${ }^{3}$ (D), Ahmed G. Abo-Khalil ${ }^{3,4}$ and \\ Guftaar Ahmad Sardar Sidhu 2,* \\ 1 Department of Physics, Govt. Sadiq College Women University, 63100 Bahawalpur, Pakistan; \\ amnamalik04@yahoo.com \\ 2 Department of Electrical Engineering, COMSATS University Islamabad, 45500 Islamabad, Pakistan; \\ zainalihanan1@gmail.com \\ 3 Department of Electrical Engineering, College of Engineering, Majmaah University, \\ 15341 Al Majmaah, Saudi Arabia; a.awan@mu.edu.sa (A.B.A.); a.abokhalil@mu.edu.sa (A.G.A.-K.) \\ 4 Electrical Engineering Department, Faculty of Engineering, Assiut University, University Street, \\ Assiut 71515, Egypt \\ * Correspondence: guftaarahmad@comsats.edu.pk; Tel.: +92-51-9049254
}

Received: 14 April 2018; Accepted: 22 May 2018; Published: 28 May 2018

\begin{abstract}
In this paper, we study the energy management techniques in the smart grid with multiple energy providers. We seek to minimize the electricity cost. In this paper, the desired objectives are achieved through scheduling of different consumers to different utilities at different time slots. We consider a practical system where multiple users can be allocated to a single utility, but, a user cannot be assigned to more than one utility. As a first goal, we formulate a sum cost minimization problem subject to independent generation capacity of each utility. A dual decomposition approach is exploited to find an efficient solution where the sub-gradient approach is adopted to update the dual variables. Later, a min-max based optimization framework is adopted to achieve the fairness among different customers. Moreover, suboptimal schemes are also designed to reduce the computational complexity. Simulation results are presented to validate the performance of the proposed solutions.
\end{abstract}

Keywords: fairness; smart grid; multiple suppliers; consumer scheduling; cost minimization

\section{Introduction}

The smart grid (SG) has emerged as one of the most exciting technologies of this century [1,2]. In contrast to conventional grid whose sole purpose was to transfer power from centralized generation to far-away distributed consumers, SG employs two-way flow of information along with providing electricity to the end users [3]. The SG benefits from communication technologies to improve security, facilitate consumer choice, and to provide power reliability [4]. Further, it enhances the coordination and the connectivity among energy providers and consumers [5]. A lot of work has been done in literature to make SG practical. A moving average based forecast model was proposed by authors in [6]. The model is useful for forecasting electric power demand from short to medium term horizon. The authors in [7] presented a analytical target cascading based distributed solution for power flow at transmission system operator and distribution system operator. The work in [8] studied the impact of uncertainty in pricing on energy hubs. A real time energy management problem was formed and the existence of unique Nash equilibrium was proven.

Demand side management (DSM) is used to control the energy consumption at customer side and is considered to be an indispensable component for deployment of future SG [9]. It encourages the customers to shift their loads in favor of reducing the overall electricity cost [10,11]. The DSM programs 
help to manage the power markets in efficient manner and are categorized into two protocols namely real time pricing (RTP) and Direct load control (DLC) [12]. In RTP programs, users can shift their loads according to different electricity prices at different hours. Thus, the users can benefit from shifting the loads to the hours with less per unit price. On the other hand, DLC programs are established by an agreement between the user and the utility company such that the electricity provider can control the energy consumption of different appliances e.g., refrigerators, air conditioners, etc., in the peak hours of consumption [13-16].

Intelligent energy management aims to achieve balance between the generation and the demand [17]. All DSM programs optimize the energy scheduling to minimize the total cost and to reduce ratio of peak usage to the average consumption i.e., peak to average ratio (PAR) [18]. A number of the DSM programs emphasized on cooperation between the users and the utility with DLC model [19]. Under the limited generation capacity and different preferences of customers, the work in [20] proposed a scheduling strategy under per hour constraints that results in different prices for different hours. In [21], authors proposed a load billing scheme that helped the individuals to reduce their energy consumption costs by scheduling their future energy consumption profiles. Real time pricing along with block rate model can be utilized to minimize electricity price and PAR [22]. The authors in [23] worked on a scheduling algorithm under water filling strategy. The results provide a constant demand behaviour over all scheduling hours. To reduce PAR, M. Rad proposed an autonomous DSM algorithm where customers can schedule their energy consumption profiles and energy payments [24]. To minimize the total cost of electricity, the authors in [25] modeled a non cooperative energy consumption game. The authors in [26], presented various techniques based on Genetic Algorithm (GA) to obtain a constant demand curve for each user. In [27], an online algorithm was proposed to attain energy scheduling where the utility aimed to minimize the overall cost of the consumers. The work in [28] considered a min-max scheduling algorithm, that was implemented to reduce PAR and energy consumption cost while keeping energy consumption profiles confidential. A time varying pricing-based DSM algorithm was suggested in [29]. Where, at first, reduction of electricity bill was accomplished and secondly load balancing algorithm was used to cut down PAR. Along with these two objectives, user convenience was also considered as a dominant issue.

\subsection{Related Work}

All the literature work discussed above had an emphasis on energy scheduling without considering fairness among consumers. Different electricity users in a particular environment may demand for fair energy scheduling [30]. The optimization solutions obtained for sum utility maximization (minimiztion) may result in unfair behaviour towards different users [31]. Thus, the energy scheduling algorithms under user fairness consideration in SG are required [32]. The authors in [33] proposed a fairness demand response (FDR) scheme to consider the satisfaction of individual users and the energy provider. Later, an autonomous demand response (DR) model is proposed in [34] to design a fair billing mechanism for different users. The users are rewarded fairly based on their contributions in achieving the system objectives. Recently, fair DR model for residential users is proposed in [35]. A strategical game theoretic model is proposed to achieve the fairness with low PAR and the solution is obtained from correlated equilibrium approach.

The works $[9,35]$ designed the energy scheduling algorithms while a a single energy provider is considered in the system. The modern SG systems may consist of multiple energy providers [36]. The techniques developed under single utility provider scenario may become invalid for multi-utility case. Thus, the study of energy scheduling under multiple energy providers becomes necessary. The work in [37] investigated the DSM techniques in the future SG under the consideration of multiple energy suppliers. The authors focused on reducing peak load on SG along with maximizing suppliers' profit and customer's payoff. The proposed algorithm reduced the PAR index by shifting the load to off peak periods. In the proposed system each supplier submits its bid to the DSM center, in response to this, the DSM center sets the price of electricity that is based on all the submitted bids. The work in [38] 
focused on a community energy storage (CES)-based SG where all the users have accurate forecast of their next day's power generation and energy requirement. This forecast is shared with the CES by each user. The CES is capable of exchanging energy with the grids as well as with all the users, hence, acts as a central node. Authors formulated a Stackleberg game between the CES and users to minimize the total cost. In [39], authors considered a SG with multiple suppliers equipped with heterogeneous energy sources. The objective of each utility was to maximize its revenue while consumers request the energy based on their demands. A game theoretical-based hierarchical decision-making scheme was designed. Further, the work in [40] proposed an incentive-based DR model with multiple service providers and the customers. In the proposed scheme, the operators offers benefits to the suppliers which in turn enrol the customers. A Stackelberg game is proposed for interaction of each energy provider with the utility. As each supplier serve only the enrolled consumers, thus, a dynamic supplier to customer assignment is missing.

\subsection{Motivation}

In DSM techniques, reported in the literature, the single utility offers different prices in different hours. In response, the consumers shift their loads to the low price hours. This reduces the overall cost of electricity at the customer end while facilitate the energy provider to reduce usage in the peak hours. In the case of multiple service providers, different prices are offered in different time slots by the suppliers. Specifically, in the competitive electricity markets, an electricity provider may offer high price in one time slot while other supplier may provide at cheap rate during that time. Under the limited capacity of each supplier and the different demands of users, scheduling the loads to reduce the electricity cost becomes a challenging problem. Further, under the dynamic customer to supplier assignment, a particular user may enjoy cheap price in all hours while minimizing the system's overall cost. Thus, the consumer fairness must be considered such that each user can benefit from comparable reduced electricity bills. However, the achieving such fairness under independent prices in different time slots by multiple energy suppliers becomes even more complex. The problem of consumer assignment to a particular energy supplier in each time slot under the limited capacity and the different load demands to reduce the sum cost and fairness among users have not been investigated in the literature, to the best of author's knowledge.

\subsection{Contributions}

In this paper, we consider a multiple energy provider (Please note that in this paper we use terms 'provider', 'supplier', and 'utility' interchangeably.) environment where different consumers are free to choose a particular utility. Each energy provider offers different prices at different times of the day and is independent of the other utilities. Our objective is to reduce the total cost of electricity as well as to minimize the electric expenditure of each user so that an efficient scheduling of consumers can be obtained. Specifically, we solve the problem that which users should be attached to which utility at different times where a supplier may not be able to serve all users in a particular time. The contributions of this work can be summarized as:

- We first formulate a consumer-to-utility assignment problem such that multiple users can be served by each utility while a particular consumer can be assigned to one and only one supplier in a given time.

- A dual decomposition-based solution is presented such that the overall cost is minimized subject to the limited available capacity of each utility. In the proposed solution, a user can choose different utilities in different time slots, thus, each utility has an independent set of users in each time.

- Later, a low complexity sub-optimal solution is proposed to minimize the sum system cost. In this algorithm, utilities are assigned to different users in sequential manner such that supplier with minimum price is selected first and users are assigned until the maximum capacity is reached. 
- Then, a fair cost minimization problem is formulated as min-max optimization under the same constraints. The complex min-max problem is first re-formulated to standard minimization problem and then duality theory is exploited to obtain the an efficient solution where dual problem is solved though sub-gradient method.

- Similar to the sum cost minimization, a low complexity algorithm for fair cost minimization is also designed.

- Finally, simulation results are presented to validate the performance of the proposed schemes.

\subsection{System Model}

We consider a smart power system with $M$ consumers and $N$ energy providers. We assume that each consumer is affixed with a smart meter that consists of Energy Consumption Scheduling (ECS) device. The smart meter is used to control user's power consumption and provide coordination between users and energy providers. All the smart meters and the energy providers are connected with the regulatory authority and communicate information over wireless transmission. This regulatory body could be considered as the dispatch center with additional smart capabilities of information transmission. We assume all the utility companies work under the control of central regulatory authority. This is a similar scenario to the mobile communication where different companies work under a regulatory body e.g., Federal Communications Commission (FCC). The considered system model is depicted below in Figure 1.

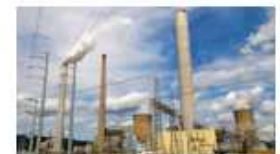

Energy Provider 1

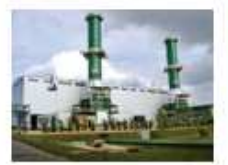

Energy Provider 2

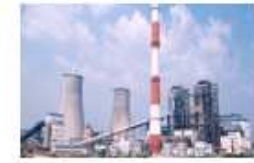

Energy Provider 3

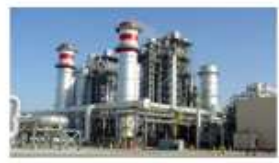

Energy Provider 4

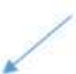

Regulatory Authority

Transmission lines
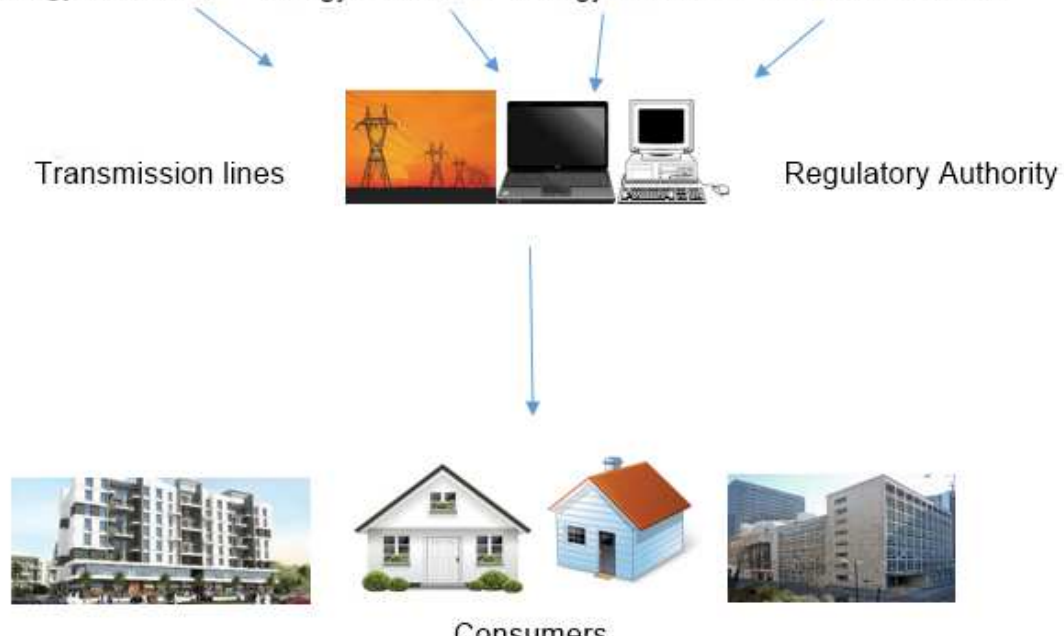

Figure 1. System Model.

\subsection{Organization of manuscript}

The rest of the paper is organized as follows: Problem formulation for total cost reduction and its solutions are presented in Section 2. Section 3 comprises of problem formulation and the corresponding solutions for fair cost minimization. Simulation results and the conclusion are given in Sections 4 and 5 , respectively. The definitions of different variables used in this paper are given in Table 1. 
Table 1. variable definitions.

\begin{tabular}{cc}
\hline$P_{i, j}$ & Electricity price of $i$ th energy provider in $j$ th hour. \\
\hline$\pi_{(k, i, j)}$ & Binary variable associated to the allocation of $k$ th user to $i$ th utility in $j$ th hour. \\
$l_{k, j}$ & Energy consumption of $k$ th user in $j$ th hour. \\
$L_{t h}^{i}$ & Generation capacity of $i$ th energy provider. \\
$t$ & Auxiliary variable associated to the fairness constraint. \\
$\lambda, \eta$ & Dual variables. \\
$M$ & Number of consumers in the system. \\
$N$ & Total energy providers in the system. \\
$H$ & Total number of time slots. \\
\hline
\end{tabular}

\section{Total Cost Minimization}

Advanced communication infrastructure enables an authentic two-way communication between energy provider and consumers. In current scenario of high electricity prices, it has become mandatory to introduce techniques and innovative methods to minimize cost of electricity. Solution to this alarming problem is possible by modern communication technology. Considering multiple energy providers, we formulate an optimization problem for sake of reducing the sum cost of electricity. In this section the discourse is around such an energy management technique in which consumer can be attached to $i$ th energy provider in $j$ th hour as prices are decided ahead. This scheduling of energy provider can reduce total cost of electricity. For allocation of energy providers to each customer we define binary variable $\pi_{(k, i, j)}$, such that:

$$
\pi_{(k, i, j)}=\left\{\begin{array}{l}
1, \text { if } k \text { th user is assigned to } i \text { thutility in } j \text { th hour } \\
0, \text { otherwise }
\end{array} .\right.
$$

With this the problem is mathematically described as:

$$
\begin{aligned}
\min _{\pi_{(k, i, j)}} & \sum_{i=1}^{N} \sum_{k=1}^{M} \sum_{j=1}^{H} P_{(i, j)} l_{(k, j)} \pi_{(k, i, j)}, \\
\text { s.t. } & \sum_{i=1}^{N} \pi_{(k, i, j)}=1, \quad \forall k, j, \\
& \sum_{k=1}^{M} \sum_{j=1}^{H} \pi_{(k, i, j)} l_{(k, j)} \leq L_{t h}^{i}, \quad \forall i,
\end{aligned}
$$

where $P_{i, j}$ is electricity price of $i$ th energy provider in $j$ th hour, $l_{k, j}$ represents energy consumption of $k$ th user in $j$ th hour and $L_{t h}^{i}$ denotes generation capacity of $i$ th energy provider. We define the set of consumers $\mathbb{K}=\{1,2,3 \ldots . M\}$, set of energy providers $\mathbb{I}=\{1,2,3 \ldots . N\}$ and set of time slots $\mathbb{J}=\{1,2,3 \ldots . H\}$, with $k \in \mathbb{K}$, where $i \in \mathbb{I}$, and $j \in \mathbb{J}$. The first constraint is showing the relation between energy provider and consumer. The $k t h$ user can be attached to one and only one energy provider in $j$ th hour. While, the second constraint represents that energy consumption of consumers attached to $i$ th energy provider must be less than the generation capacity of that energy source. 


\subsection{Proposed Scheme}

The formulated problem is a binary integer programming problem [41]. To efficiently solve the optimization problem, the corresponding dual problem is given as:

$$
\begin{array}{cl}
\max _{\lambda_{i}} & D\left(\lambda_{i}\right), \\
\text { s.t. } & \lambda_{i} \geq 0,
\end{array}
$$

where:

$$
\begin{aligned}
D\left(\lambda_{i}\right)= & \min _{\pi_{(k, i, j)}} L\left(\pi_{(k, i, j)}, \lambda_{i}\right) . \\
\text { s.t. } & \sum_{i=1}^{N} \pi_{(k, i, j)}=1, \forall k \in \mathbb{K}, j \in \mathbb{J} .
\end{aligned}
$$

The $L\left(\pi_{(k, i, j)}, \lambda_{i}\right)$ represents Lagrangian associated with the optimization problem and is defined as:

$$
L\left(\pi_{(k, i, j)}, \lambda_{i}\right)=\sum_{i=1}^{N} \sum_{k=1}^{M} \sum_{j=1}^{H} P_{(i, j)} l_{(k, j)} \pi_{(k, i, j)}+\sum_{i=1}^{N} \lambda_{i}\left(\sum_{k=1}^{M} \sum_{j=1}^{H} l_{(k, j)} \pi_{(k, i, j)}-L_{t h}^{i}\right),
$$

where $\lambda_{i}$ is known as Lagrangian multiplier. With further simplification, we obtain:

$$
L\left(\pi_{(k, i, j)}, \lambda_{i}\right)=\sum_{i=1}^{N} \pi_{(k, i, j)}\left(\sum_{k=1}^{M} \sum_{j=1}^{H}\left(P_{(i, j)} l_{(k, j)}+\lambda_{i} l_{(k, j)}\right)\right)-\sum_{i=1}^{N} \lambda_{i} L_{t h}^{i}
$$

Thus, the optimization in (5) is equivalent to following optimization

$$
\begin{aligned}
& \min _{\pi_{(k, i, j)}} \sum_{k=1}^{M} \sum_{j=1}^{H} P_{(i, j)} l_{(k, j)}+\lambda_{i} l_{(k, j)}, \\
& \text { s.t. } \quad \sum_{i=1}^{N} \pi_{(k, i, j)}=1, \forall k \in \mathbb{K}, j \in \mathbb{J} .
\end{aligned}
$$

This standard assignment problem and the solution $\pi_{(k, i, j)}^{*}$ of this problem is:

$$
\pi_{(k, i, j)}^{*}=\arg \min _{i} P_{(i, j)} l_{(k, j)}+\lambda_{i} l_{(k, j)}, \forall k \in \mathbb{K}, j \in \mathbb{J} .
$$

The dual variable $\lambda_{i}$ is obtained through an iterative method [42], such that at $(n+1)$ th iteration $\lambda_{i}$ is updated as

$$
\lambda_{i}(i t r+1)=\lambda_{i}(i t r)+\delta_{i}(i t r)\left(\sum_{k=1}^{M} \sum_{j=1}^{H} \pi_{(k, i, j)} l_{k, j}-L_{t h}^{i}\right)
$$

where $\delta_{i}(i t r)$ is the step size for $i$ th update at iteration $i t r$, the dual variable is updated until convergence. Thus the optimized solution is found from described method.

\subsection{Suboptimal Scheme for Total Cost Minimization}

The algorithm described in the previous section can efficiently reduce the sum cost of electricity but when number of consumers are increased solution becomes complex. To reduce the computational 
complexity of the solution, we propose a sub-optimal scheme. The algorithm first selects the $i$ th utility that provides minimum price. Users are attached to that utility until its generation capacity is reached. When the selected utility is unable to serve more consumers second utility with minimum price is selected and this process is repeated for all the remaining utilities. It involves the steps given in Algorithm 1.

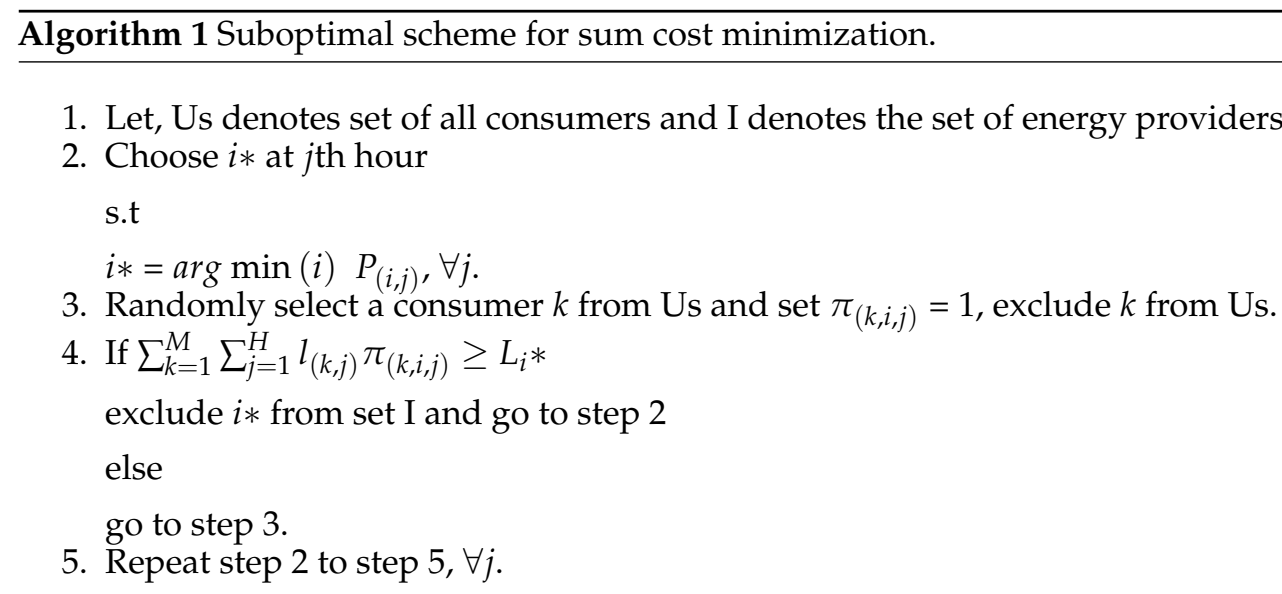

\section{Fair Cost Minimization}

So far our work circled around overall cost minimization of electricity without considering consumer fairness i.e., some users have less electricity charges and others getting high charges. Now our focus is on consumer impartiality. We propose an algorithm which aims that consumers will have no discrimination of electricity charges among them. The problem can be stated mathematically as:

$$
\begin{aligned}
\min _{\pi_{(k, i, j)}} \max _{k} & \sum_{i=1}^{N} \sum_{j=1}^{H} P_{(i, j)} l_{(k, j)} \pi_{(k, i, j)} \\
\text { s.t. } & \sum_{i=1}^{N} \pi_{(k, i, j)}=1, \quad \forall k, j, \\
& \sum_{k=1}^{M} \sum_{j=1}^{H} \pi_{(k, i, j)} l_{(k, j)} \leq L_{t h}^{i} \quad \forall i .
\end{aligned}
$$

To find the solution of problem, we first change the problem into a standard optimization problem. Introducing an auxiliary variable $t$, we can reformulate the problem as:

$$
\begin{array}{ll} 
& \min _{\pi_{(k, i, j)}, t} \\
\text { s.t. } & t \geq \sum_{i=1}^{N} \sum_{j=1}^{H} P_{(i, j)} l_{(k, j)} \pi_{(k, i, j)}, \quad \forall k, \\
& \sum_{i=1}^{N} \pi_{(k, i, j)}=1, \quad \forall k, j, \\
& \sum_{k=1}^{M} \sum_{j=1}^{H} \pi_{(k, i, j)} l_{(k, j)} \leq L_{t h}^{i} \quad \forall i .
\end{array}
$$




\subsection{Proposed Solution}

For the problem in (1), the dual function is defined as:

$$
D\left(\lambda_{k}, \eta_{i, j}\right)=\min _{\pi_{(k, i, j)}, t} L\left(\pi_{(k, i, j)}, \lambda_{k}, \eta_{i, j}, t\right) .
$$

The dual problem is given as:

$$
\begin{array}{ll}
\max _{\lambda_{k}, \eta_{i, j}} & D\left(\lambda_{k}, \eta_{i, j}\right), \\
\text { s.t. } & \lambda_{k} \geq 0, \quad \eta_{i, j} \geq 0 .
\end{array}
$$

For $t \geq 0$ minimizing $t$ is equivalent to minimizing $t^{2}$, then the Lagrangian $\mathrm{L}$ can be written as:

$$
L=t^{2}+\sum_{k=1}^{M} \lambda_{k}\left(\sum_{i=1}^{N} \sum_{j=1}^{H} P_{(i, j)} l_{(k, j)} \pi_{(k, i, j)}-t\right)+\sum_{i=1}^{N} \sum_{j=1}^{H} \eta_{i, j}\left(\sum_{k=1}^{M} \pi_{(k, i, j)} l_{(k, j)}-L_{t h}^{i}\right) .
$$

After converting original problem to sub problems for the ease of solution we get:

$$
\min _{t}\left(t^{2}-t+\sum_{k=1}^{M} \lambda_{k}\right)
$$

and

$$
\min _{\pi_{(k, i, j)}} \sum_{k=1}^{M} \lambda_{k}\left(\sum_{i=1}^{N} \sum_{j=1}^{H} P_{(i, j)} l_{(k, j)} \pi_{(k, i, j)}\right)+\sum_{i=1}^{N} \sum_{j=1}^{H} \eta_{i, j}\left(\sum_{k=1}^{M} \pi_{(k, i, j)} l_{(k, j)}-L_{t h}^{i}\right) .
$$

Solving the sub-problem (21) we obtain:

$$
t=1 / 2 \sum_{k=1}^{M} \lambda_{k}
$$

Sub-problem (22) can be simplified as:

$$
\min _{\pi_{(k, i, j)}} \sum_{i=1}^{N} \sum_{j=1}^{H} \sum_{k=1}^{M} \pi_{(k, i, j)}\left(l_{(k, j)}\left(\lambda_{k} P_{(i, j)}+\eta_{i, j}\right)\right) .
$$

Thus the optimum value of $\pi_{(k, i, j)}$ is:

$$
\pi_{(k, i, j)}^{*}=\left\{\begin{array}{l}
1, \text { for } k=\arg \min _{i}\left(l_{(k, j)}\left(\lambda_{k} P_{(i, j)}+\eta_{i, j}\right)\right) \\
0, \text { otherwise, } \quad \forall j \in \mathbb{J} .
\end{array}\right.
$$

Dual problem can be solved by iterative method to attain guaranteed convergence. While, dual variables are obtained from following updates:

$$
\begin{aligned}
& \lambda_{k}(i t r+1)=\lambda_{k}(i t r)+\delta_{1}(i t r)\left(\sum_{i=1}^{N} \sum_{j=1}^{H} P_{(i, j)} l_{(k, j)} \pi_{(k, i, j)-t}\right), \\
& \eta_{i, j}(i t r+1)=\eta_{i, j}(i t r)+\delta_{2}(i t r)\left(\sum_{k=1}^{M} \pi_{(k, i, j)} l_{(k, j)}-L_{t h}^{i}\right) .
\end{aligned}
$$


Dual variables $\left(\lambda_{k}, \eta_{i, j}\right)$ are updated until convergence.

\subsection{Suboptimal Scheme for Fair Cost Minimization}

Similar to the previous section, a sub-optimal solution is also designed to reduce the computational complexity. In this algorithm, rather than allocating users to the utility with minimum price $\forall j$, we attach $k$ th user with the utility offering minimum price in $j$ th hour. When the maximum load limit of the cheapest utility is achieved, it is removed from the vector of available utilities (I) and the process is repeated for the next cheapest utility until all the users are attached with utilities $\forall j$. This allows more users to take advantage of the utilities providing energy at less cost and distributes the price among users. The proposed scheme is described as shown in Algorithm 2. Figure 2 shows the two scenarios where the proposed schemes can be implemented to get the desired performance.

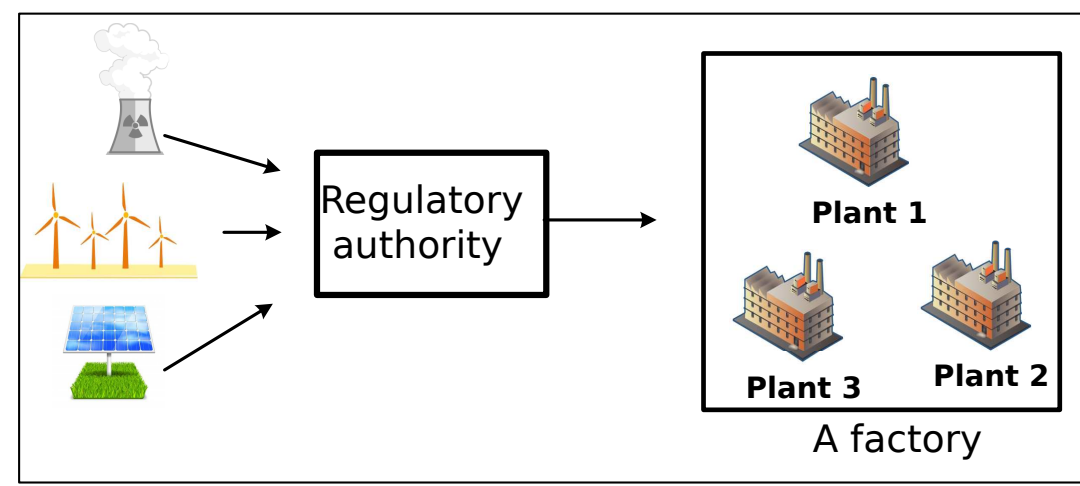

Scenario 1

Minimum cost requirement

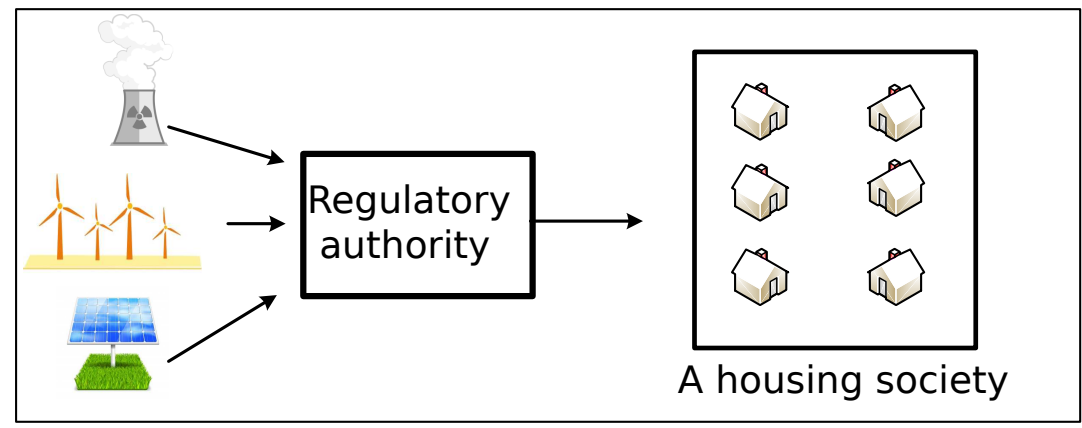

Scenario 2

Fair cost requirement

Figure 2. Example of scenarios where fairness in cost and sum cost minimization is required.

\section{Algorithm 2 Suboptimal scheme for fair cost minimization.}

1. Initiallization: $\pi_{k, i, j}=\mathbf{0}, \forall i$

2. Choose consumer $k$ from set of consumers Us.

3. Select $i *$ at $j$ th hour for $k$ th consumer

s.t

$i *=\arg \min (i) P_{(i, j)}, \forall j$.

4. Set $\pi_{k, i, j}=1$, until $\sum_{k=1}^{M} \sum_{j=1}^{H} l_{(k, j)} \pi_{(k, i, j)} \leq L_{i} *$, and eliminate $k$ from Us.

5. If $\sum_{k=1}^{M} \sum_{j=1}^{H} l_{(k, j)} \pi_{k, i, j} \geq L_{i} *$, exclude $i *$ from I and go to step 3 .

6. Repeat step 2 to step $5, \forall k$. 


\section{Simulation Results}

In this section, we evaluate the performances of our purposed algorithms. We take 50 consumers and four energy providers and consider the scheduling in an entire day i.e., we set $M=50, N=4$ and $H=24$. Results for following three schemes are presented.

- OptSol: This refers to the proposed solutions in Sections 2.1 and 3.1.

- SubOptSol: Low complexity sub-optimal schemes proposed in Sections 2.2 and 3.2.

- NonOptSol: In this solution, users are selected randomly and are assigned to a randomly selected utility for the entire time horizon. This allocation stay continued until utility is unable to serve more consumers. Then another utility is selected and this scenario is continued until all the consumers are scheduled for all time slots.

\subsection{Simulation Results For Total Cost Minimization}

In this subsection, we present the simulation results for the first scenario where the objective is to minimize the total cost among all consumers.

Figure 3 shows the simulation results in terms of reducing sum cost, while consumers are assigned with different energy providers in different hours. It can be seen that our proposed optimized scheme always gives less cost and outperforms the SubOptSol and NonOptSol schemes. We can observe that for increasing the number of consumers difference between the OptSol and NonOptSol schemes is increasing. Further, the performance gap between OptSol and SubOptSol also becomes obvious for higher number of consumers and the difference increases with the increasing users. Thus, it can be inferred that our proposed OptSol can deliver more effectual results for practical number of power costumers. Last but not least, the proposed sub-optimal solution also yields significant advantage over the NonOptSol and can be adopted for higher benefits at the cost of small performance degradation as compared to the proposed OptSol.

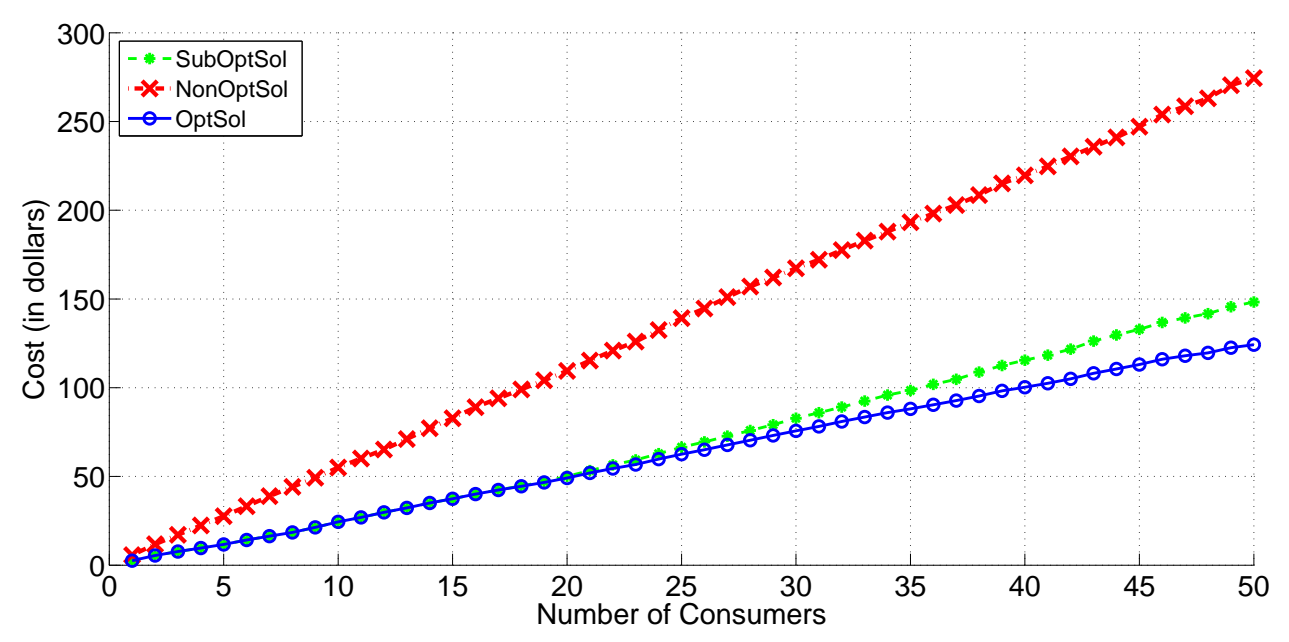

Figure 3. Sum Cost Minimization: Number of Consumers v/s Cost.

Next we analyze the impact of varying number of energy providers for $N=50$ in Figure. 4. First, we observe that the proposed scheme out performs the other candidates. However, the difference with the NonOptSol is much higher than the SubOptSol. The NonOptSol yields almost the constant results. This is because we assume that each utility provides a near feasible solution, i.e., it can accommodate maximum number of users. Thus, the significant of multiple utilities without looking into price differences in each time slot becomes invalid. For the OptSol and the SubOptSol, the cost is inversely proportional to the number of energy provides. This is because the higher number of utilities provide more opportunities in the optimization for per time slot assignment to minimize the total cost i.e., 
availability of multiple energy sources with different offered prices in each time slot provide more flexibility in scheduling.

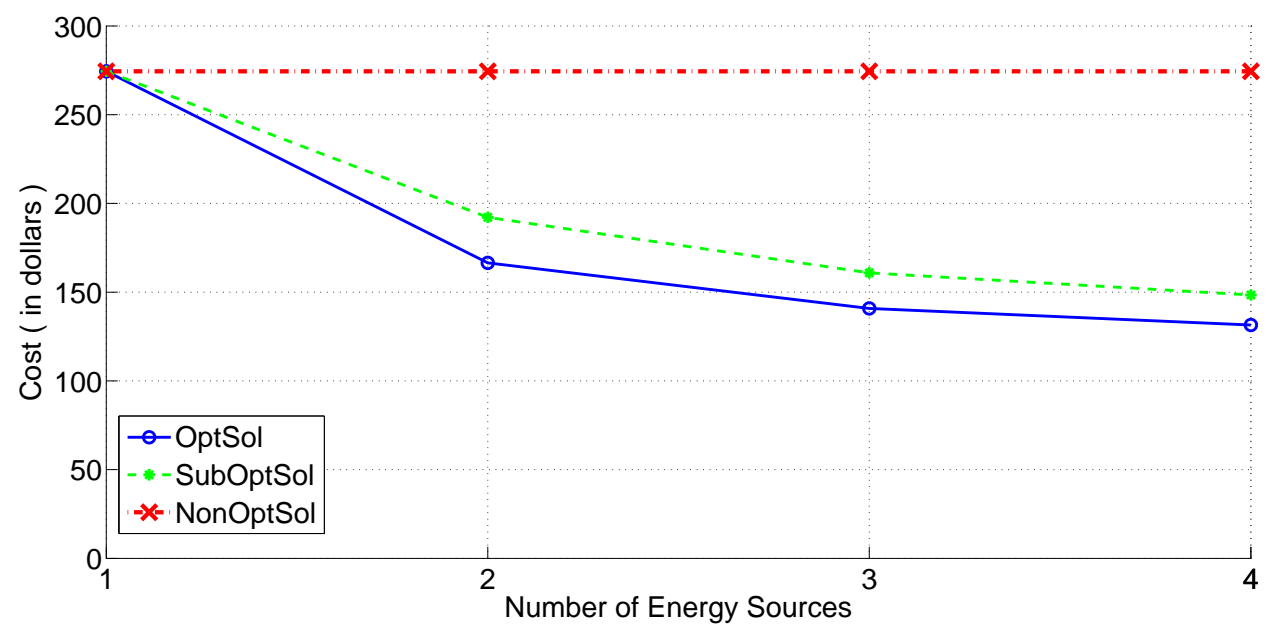

Figure 4. Sum Cost Minimization versus Number of Energy Sources.

Finally to emphasize on the feasibility of the proposed solution in terms of computational complexity, we plot the convergence behavior of the dual variables in Figure 5. It can be observed that dual variables converge within reasonable number of iterations. Thus, we conclude that our proposed optimization solution provides significant cost reduction at an acceptable convergence time.

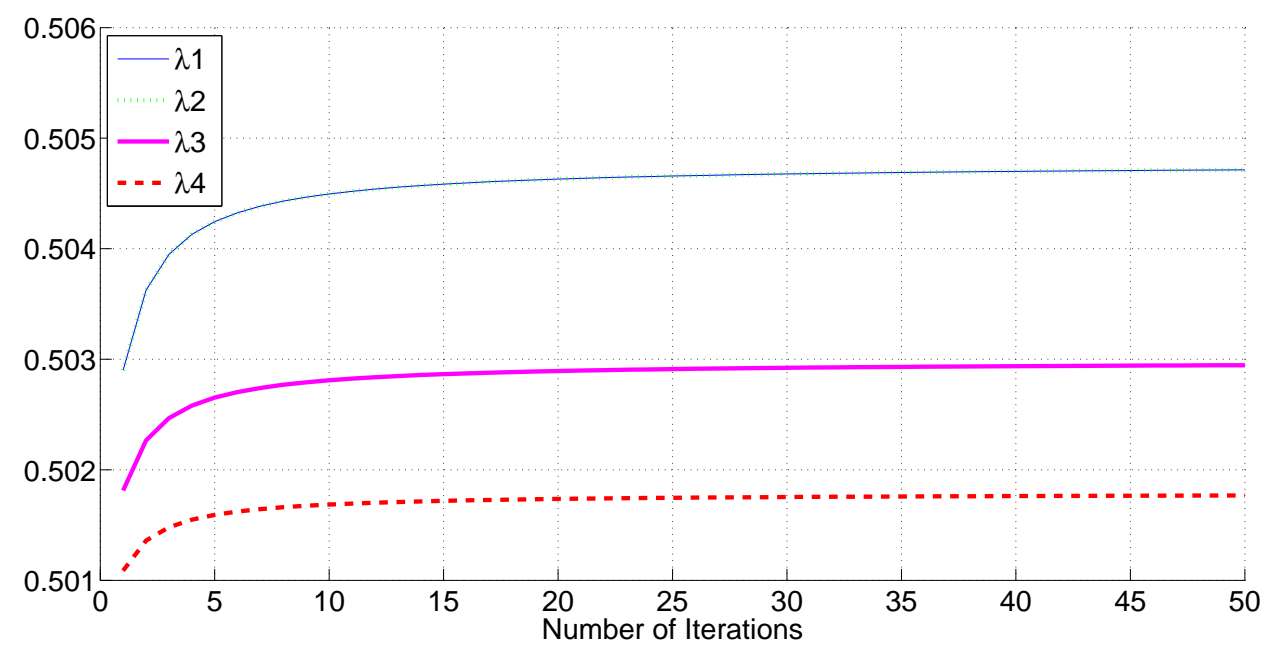

Figure 5. Convergence of Dual Variables.

\subsection{Simulation Results For Fair Cost Minimization}

The simulation results in this section are meant to demonstrate the efficacy of scheme purposed in Section 3.

Figure 6 shows that the fairness among each user is obtained. The cost difference among users is reduced and nearly flattened curve is obtained. Almost all the consumers are getting equal prices that confiscates inequity and discrimination among them. We can observe that there is a significant difference among OptSol and NonOptSol schemes. Clear peaks are observed in NonOptSol scheme due to different electricity charges for users. Close results are obtained for OptSol and SubOptSol schemes as both tried to attach to the utility that provides minimum price in that hour. Thus, it clearly exhibits that OptSol technique surpasses the other schemes. 


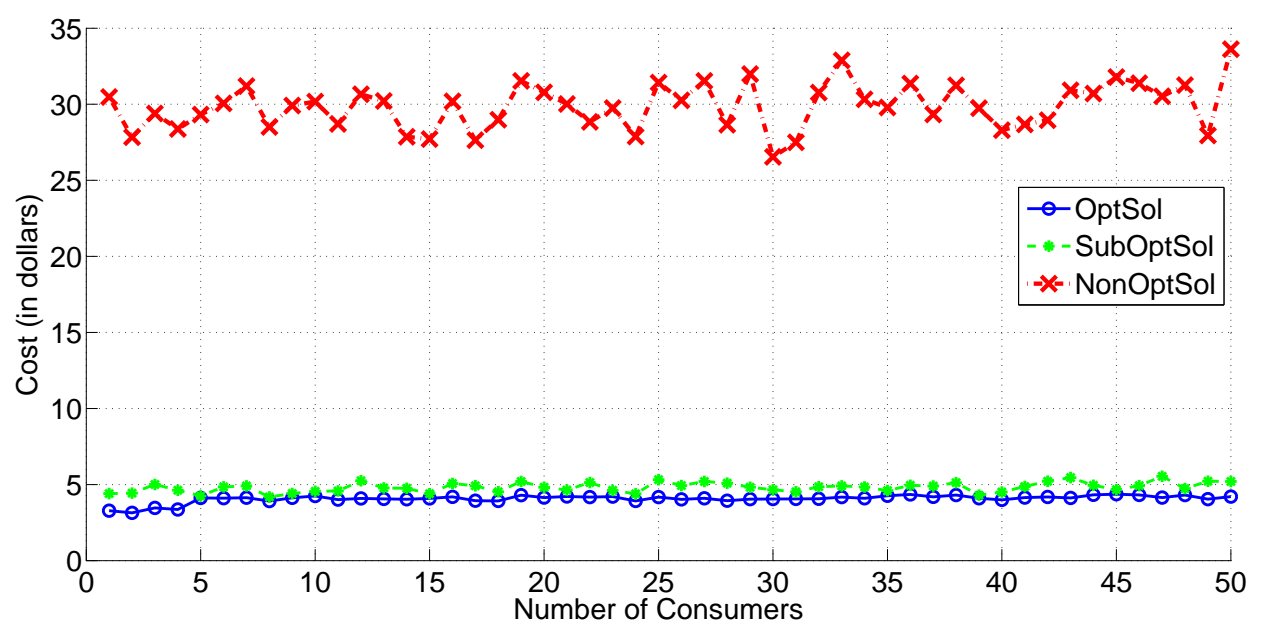

Figure 6. Fair Cost Minimization For 50 Consumers.

Figure 7 and 8 represent $k$ th user cost with different energy sources. It can be seen that the performance of schemes improves with increasing number of energy providers. Therefore, by increasing energy sources fairness is achieved along with cost reduction, that is beneficial for consumers as it is their utmost desire.
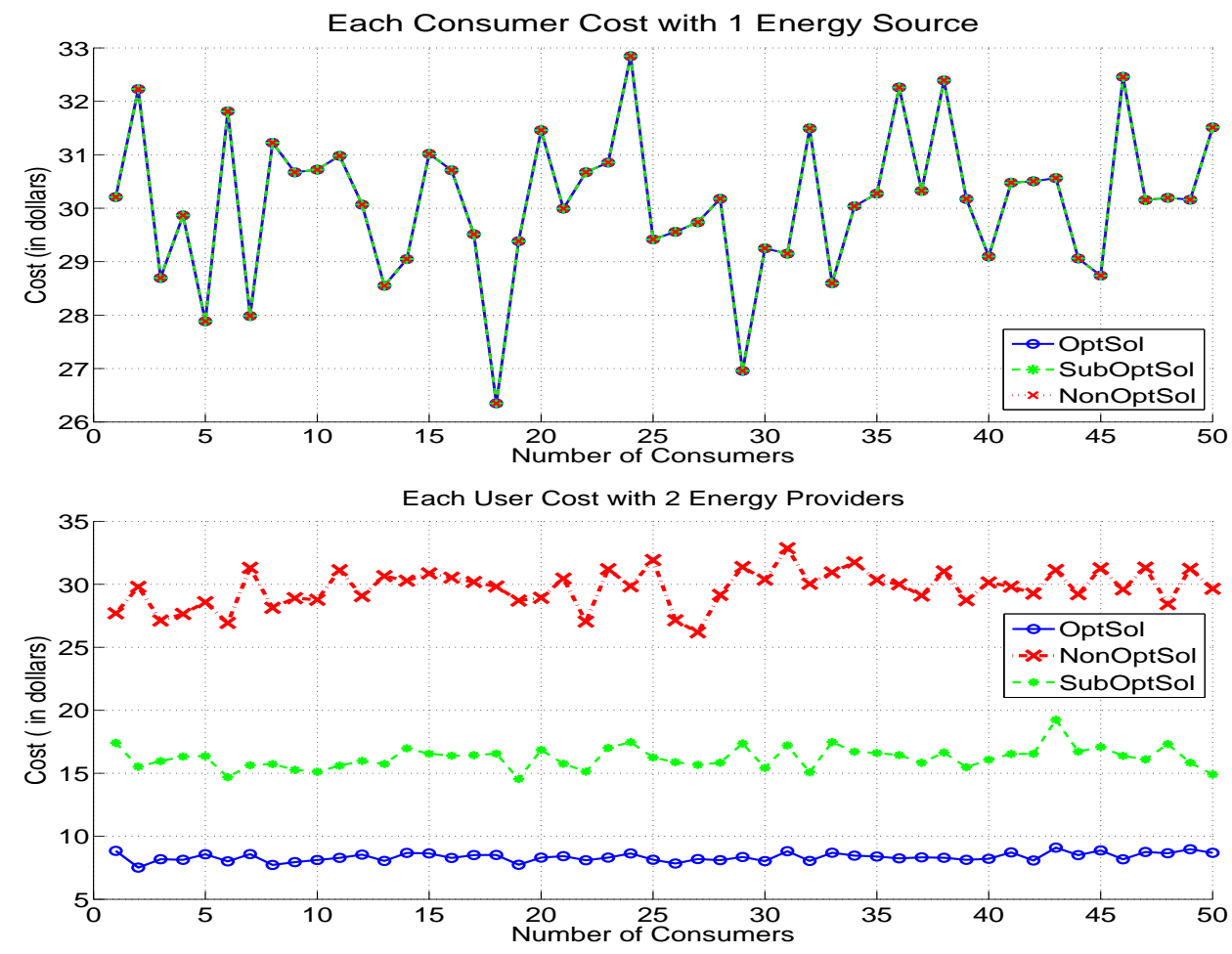

Figure 7. Fair Cost Minimization versus Number of Energy Sources for $N=1$ and $N=2$. 

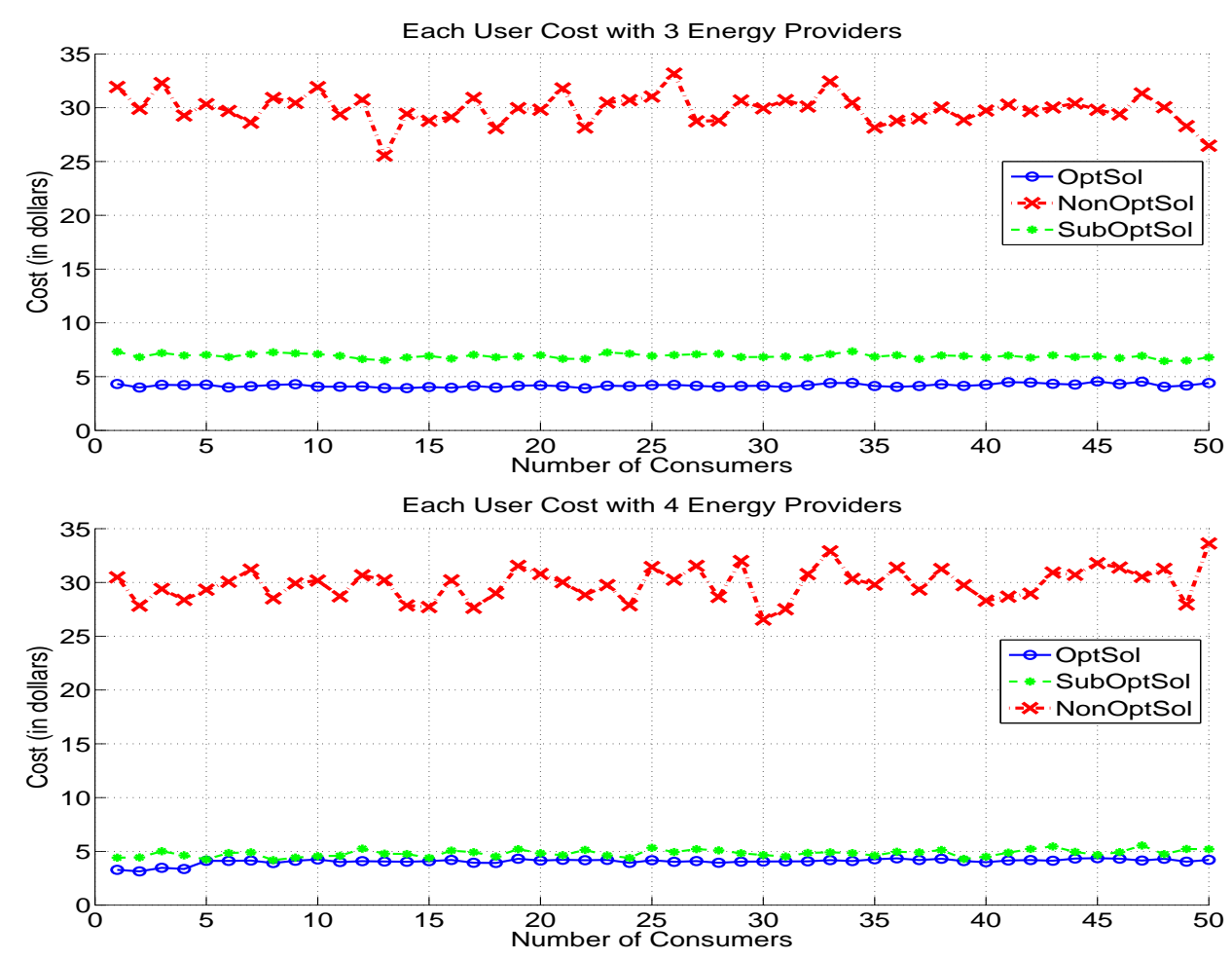

Figure 8. Fair Cost Minimization versus Number of Energy Sources for $N=3$ and $N=4$.

Figure 9 illustrates the number of iterations needed for the convergence of dual variables. Convergence is attained on only small number of iterations. Further, all the simulation results shown above validate the performance of proposed work.
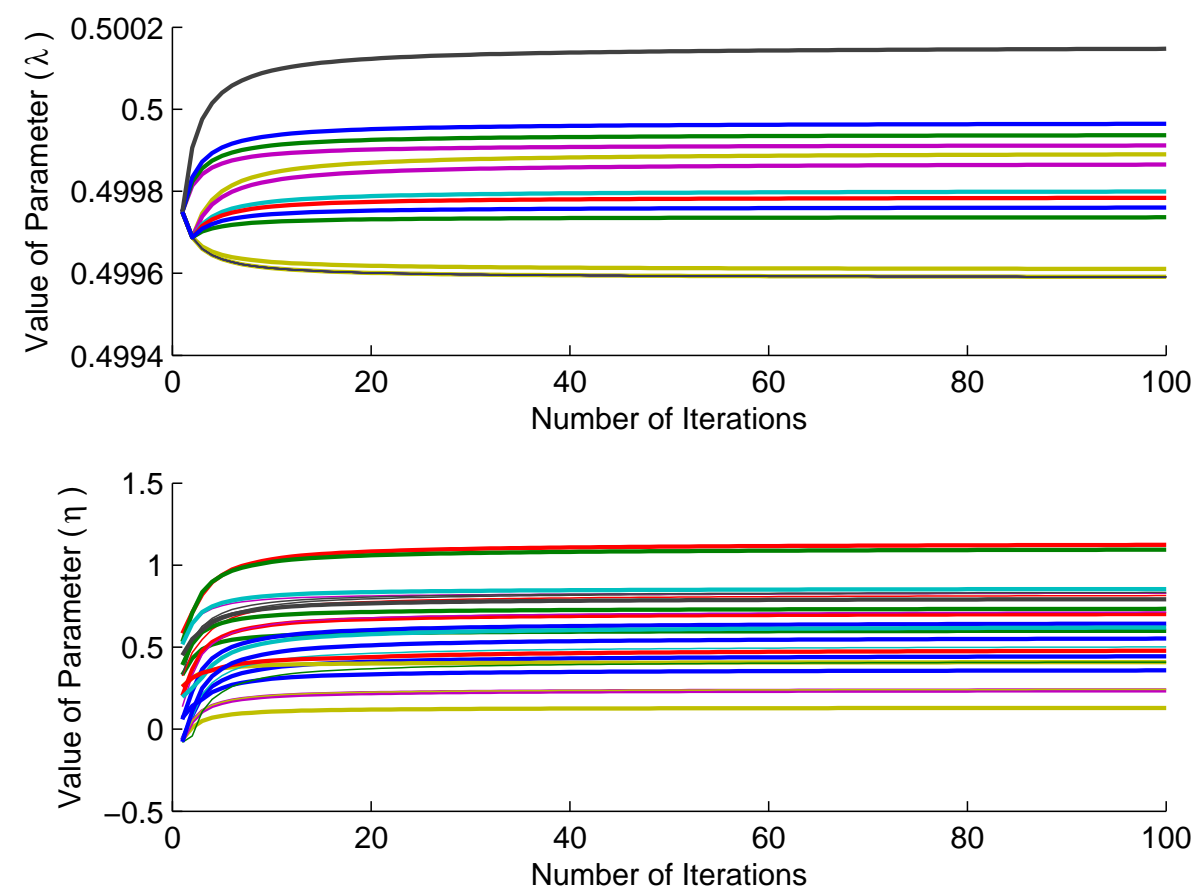

Figure 9. Convergence versus No of Iterations. 
In Figure 10 and 11, we look into the relationship of both purposed algorithms, total cost minimization without fairness (WOF) and fair cost minimization (WF). We observe that there is always a trade off between both schemes. Achieving fairness among consumers increases the sum cost. On the other hand, we notice that sum cost is minimized while compromising on user fairness concerns.

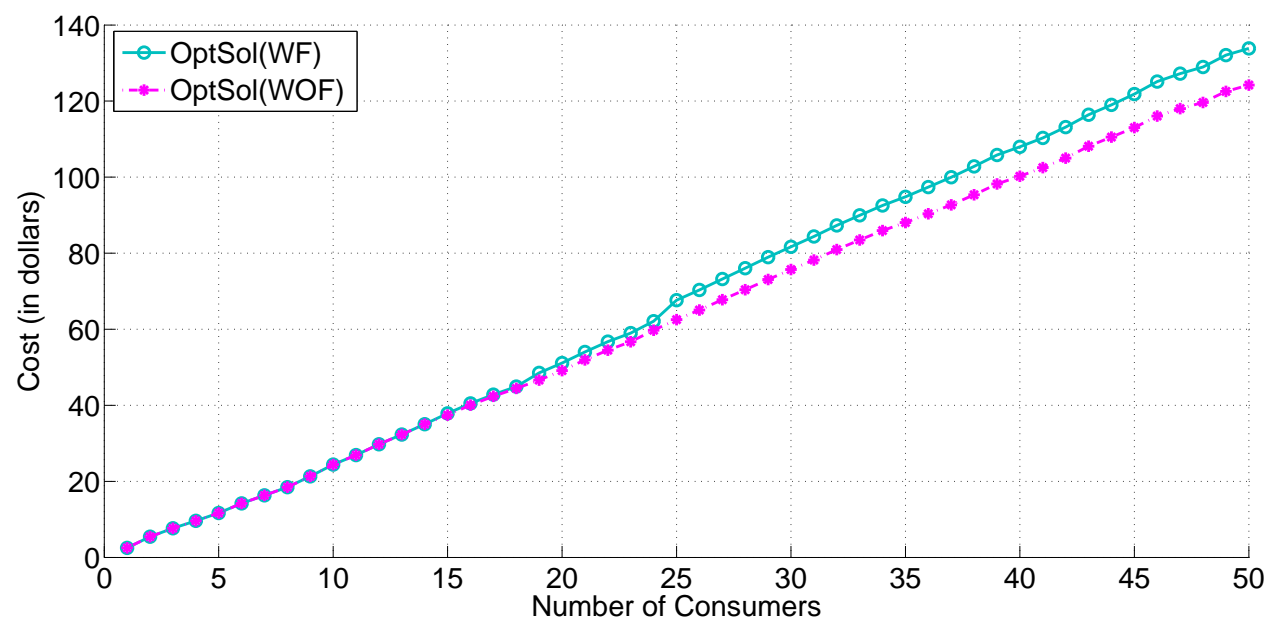

Figure 10. Sum Cost Comparison of both Proposed Schemes.

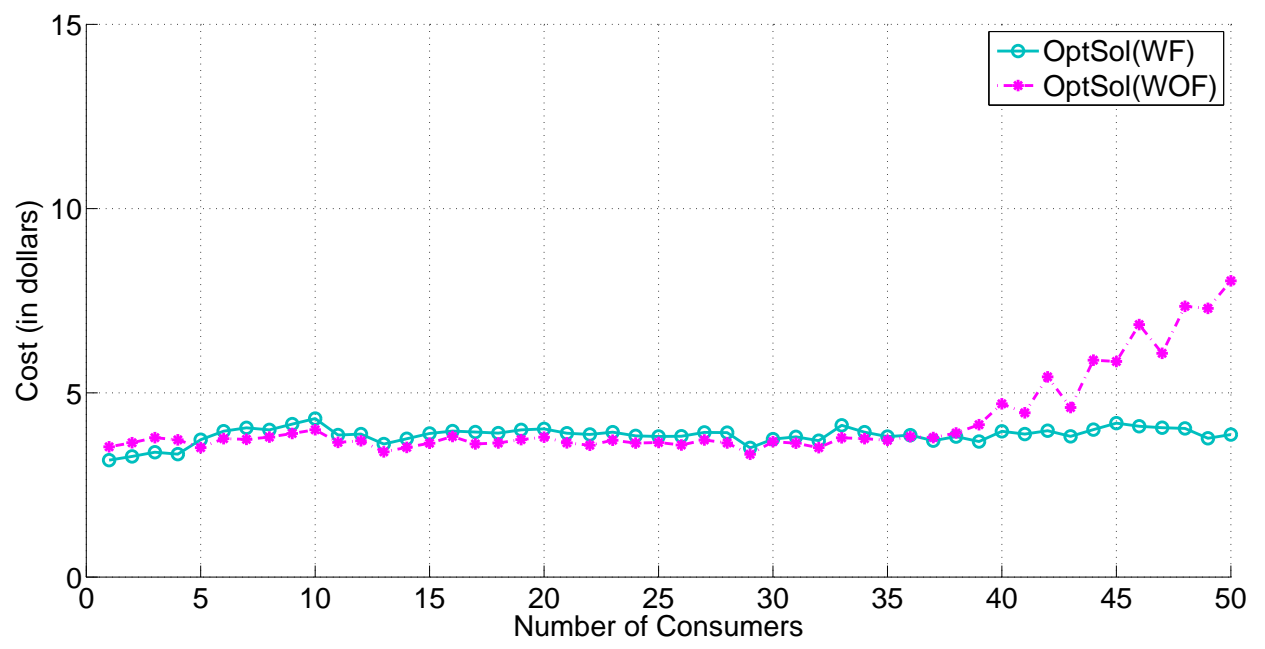

Figure 11. Cost Comparison of both Proposed Schemes.

\section{Conclusions}

This work considered multiple energy providers to meet the energy demands of several consumers. We have proposed user assignment schemes and the target was to allocate utilities to users to minimize total electricity cost where s user can switch from one utility to any other in different time slots. Both the joint optimal and the less complex sub-optimal scheme ware presented. We have evaluated the performances of both schemes through simulation results. Further, to impart fairness among consumers in terms of their electricity cost, our second goal was to reduce the difference in the electricity cost among different users. Dual decomposition-based solutions are obtained where primal variables are obtained which minimized the dual function while the dual variables are updated from sub-gradient method. At the end, we have compared all schemes through simulation results. The results validated the proposed solutions. In the future work, the more practical constraints of the power system could be included in the proposed framework. Further, the renewable source 
can be integrated in the system model which will bring many exciting benefits at the cost of more challenging problem.

Author Contributions: All authors have equal contributions.

Funding: This research was funded by the Deanship of Scientific Research (DSR) at Majmaah University, Majmaah Saudi Arabia, through vote number $68 \backslash 37$.

Acknowledgments: The authors acknowledge with thanks the Deanship of Scientific Research (DSR) at Majmaah University, Majmaah Saudi Arabia, the Department of Electrical Engineering, COMSATS University Islamabad, and the Higher Education Commission (HEC) of Pakistan for providing facilities for this research project.

Conflicts of Interest: The authors declare no conflict of interest.

\section{References}

1. Bahrami, S.; Wong, V.W.S. An autonomous demand response program in smart grid with foresighted users. In Proceedings of the 2015 IEEE International Conference on Smart Grid Commun. (SmartGridComm), Miami, FL, USA, 2015; pp. 205-210.

2. Amini, M.H.; Nabi, B.; Haghifam, M.R. Load management using multi-agent systems in smart distribution network. In Proceedings of the IEEE Power \& Energy Society General Meeting, Vancouver, ON, Canada, 21-25 July 2013; pp. 1-5.

3. Farhangi, H. The path of the smart grid. IEEE Power Energy Mag. 2010, 8, 18-28. [CrossRef]

4. Gharavi, H.; Ghafurian, R. Smart grid: The electric energy system of the future. IEEE 2011, 99, 917-921. [CrossRef]

5. Xi, F.; Satyajayant, M.; Guoliang, X.; Dejun, Y. Smart grid—The new and improved power grid: A survey. IEEE Commun. Surv. Tutor. 2011, 14, 1-37.

6. Boroojeni, K.G.; Amini, M.H.; Bahrami, S.; Iyengar, S.S.; Sarwat, A.I.; Karabasoglu, O. A novel multi-timescale modeling for electric power demand forecasting: From short-term to medium-term horizon. Electr. Power Syst. Res. 2017, 142, 58-73. [CrossRef]

7. Mohammadi, A.; Mehrtash, M.; Kargarian, A. Diagonal quadratic approximation for decentralized collaborative TSO+DSO optimal power flow. IEEE Trans. Smart Grid 2018. [CrossRef]

8. Bahrami, S.; Toulabi, M.; Ranjbar, S.; Aghtaie, M.M.; Ranjbar, A.M. A decentralized energy management framework for energy hubs in dynamic pricing markets. IEEE Trans. Smart Grid 2017. [CrossRef]

9. Palensky, P.; Dietrich, D. Demand side management: Demand response, intelligent energy systems, and smart loads. IEEE Trans. Ind. Inform. 2011, 7, 381-388. [CrossRef]

10. Ramanathan, B.; Vittal, V. A framework for evaluation of advanced direct load control with minimum disruption. IEEE Trans. Power Syst. 2008, 23, 1681-1688. [CrossRef]

11. Basit, A.; Sidhu, G.A.S.; Mahmood, A.; Gao, F. Efficient and autonomous energy management techniques for the future smart homes. IEEE Trans. Smart Grid 2017, 8, 917-926. [CrossRef]

12. Gellings, C.W.; Chamberlin, J.H. Demand Side Management: Concepts and Methods, 2nd ed.; PennWell Books: Tulsa, OK, USA, 1993.

13. Weers, D.D.; Shamsedin, M.A. Testing a new direct load control power line communication system. IEEE Trans. Power Del. 1987, 2, 657-660. [CrossRef]

14. Chu, C.M.; Jong, T.L.; Huang, Y.W. A direct load control of air-conditioning loads with thermal comfort control. In Proceeding of the IEEE Power Engineering Society General Meeting, San Francisco, CA, USA, 16 June 2005; pp. 664-669.

15. Gomes, A.; Antunes, C.H.; Martins, A.G. A multiple objective approach to direct load control using an interactive evolutionary algorithm. IEEE Trans. Power Syst. 2007, 22, 1004-1011. [CrossRef]

16. Jindal, A.; Kumar, N.; Rodrigues, J.J. A heuristic-based smart HVAC energy management scheme for university buildings. IEEE Trans. Ind. Inform. 2018. [CrossRef]

17. Tiptipakorn, S.; Wei-Jen, L. A residential consumer-centered load control strategy in real-time electricity pricing environment. In Proceedings of the 39th North American Power Symposium, Las Cruces, NM, USA, 30 September-2 October 2007; pp. 505-510.

18. Zhao, Z.; Lee, W.C.; Shin, Y.; Song, K.B. An optimal power scheduling method for demand response in home energy management system. IEEE Trans. Smart Grid 2013, 4, 1391-1400. [CrossRef] 
19. Ruiz, N.; Cobelo, I.; Oyarzabal, J. A direct load control model for virtual power plant management. IEEE Trans. Power Systems 2009, 24, 959-966. [CrossRef]

20. Tarasak, P. Optimal real-time pricing under load uncertainty based on utility maximization for smart grid. In Proceedings of the IEEE International Conference on Smart Grid Communications (SmartGridComm), Brussels, Belgium, 17-20 October 2011; pp. 321-326.

21. Chen, H.; Li, Y.; Louie, R.H.; Vucetic, B. Autonomous demand side management based on energy consumption scheduling and instantaneous load billing: An aggregative game approach. IEEE Trans. Smart Grid 2014, 5, 1744-1754. [CrossRef]

22. Mohsenian-Rad, A.H.; Leon-Garcia, A. Optimal residential load control with price prediction in real-time electricity pricing environments. IEEE Trans. Smart Grid 2010, 1, 120-133. [CrossRef]

23. Shinwari, M.; Youssef, A.; Hamouda, W. A water-filling based scheduling algorithm for the smart grid. IEEE Trans. Smart Grid 2012, 3, 710-719. [CrossRef]

24. Mohsenian-Rad, A.H.; Wong, V.W.; Jatskevich, J.; Schober, R.; Leon-Garcia, A. Autonomous demand-side management based on game-theoretic energy consumption scheduling for the future smart grid. IEEE Trans. Smart Grid 2010, 1, 320-331. [CrossRef]

25. Ma, K.; Hu, G.; Spanos, C.J. Energy consumption scheduling in smart grid: A non-cooperative game approach. In Proceedings of the 9th Asian Control Conference (ASCC), Istanbul, Turkey, 23-26 June 2013; pp. 1-6.

26. Imamura, A.; Yamamoto, S.; Tazoe, T.; Onda, H.; Takeshita, H.; Okamoto, S.; Yamanaka, N. Distributed demand scheduling method to reduce energy cost in smart grid. In Proceedings of the IEEE Region 10 Humanitarian Technology Conference (R10-HTC), Sendai, Japan, 26-29 August 2013; pp. 148-153.

27. Guo, Y.; Pan, M.; Fang, Y.; Khargonekar, P.P. Coordinated energy scheduling for residential households in the smart grid. In Proceedings of the IEEE Third International Conference on Smart Grid Communications (SmartGridComm), Tainan, Taiwan, 5-8 November 2012; pp. 121-126.

28. Xiao, J.; Chung, J.Y.; Li, J.; Boutaba, R.; Hong, J.W.K. Near optimal demandside energy management under real-time demand-response pricing. In Proceedings of the International Conference on Network and Service Management (CNSM), Niagara Falls, ON, USA, 25-29 October 2010; pp. 527-532.

29. Bae, H.; Yoon, J.; Lee, Y.; Lee, J.; Kim, T.; Yu, J.; Cho, S. User-friendly demand side management for smart grid networks. In Proceedings of the International Conference on Information Networking (ICOIN), Phuket, Thiland, 10-12 February 2014; pp. 481-485.

30. Assaf, T.; Osman, A.H.; Hassan, M. Fair autonomous energy consumption scheduling based on gametheoretic approach for the future smart grid. In Proceedings of the 18th International Conference on Computer Modelling and Simulation (UKSim), Cambridge, UK, 6-8 April 2016; pp. 235-239.

31. Ali, Z.; Sidhu, G.A.S.; Waqas, M.; Gao, F.; Jin, S. Achieving energy fairness in multiuser uplink CR transmission. In Proceedings of the IEEE Wireless Communication and Networking Conference, Doha, Qatar, 3-6 April 2016; pp. 1-6.

32. Lin, J.; Yu, W.; Yang, X. Towards multistep electricity prices in smart grid electricity markets. IEEE Trans. Parallel Distrib. Syst. 2016, 27, 286-302.

33. Vuppala, S.K.; Padmanabh, K.; Bose, S.K.; Paul, S. Incorporating fairness within demand response programs in smart grid. In Proceedings of the Innovative Smart Grid Technologies (ISGT), Hilton Anaheim, CA, USA, 17-19 January 2011; pp. 1-9.

34. Baharlouei, Z.; Hashemi, M.; Narimani, H.; Mohsenian-Rad, H. Achieving optimality and fairness in autonomous demand response: Benchmarks and billing mechanisms. IEEE Trans. Smart Grid 2013, 4, 968-975. [CrossRef]

35. Pal, S.; Thakur, S.; Kumar, R.; Panigrahic, B.K. A strategical game theoretic based demand response model for residential consumers in a fair environment. Int. J. Electr. Power Energy Syst. 2018, 97, 201-210. [CrossRef]

36. Loe, R.R. Multi-utility metering for residential customers in a competitive utility environment. In Proceedings of the Ninth International Conference on Metering and Tariffs for Energy Supply, Birmingham, UK, 25-28 May 1992; pp. 37-42.

37. Jalali, M.M.; Kazemi, A. Demand side management in a smart grid with multiple electricity suppliers. Energy 2015, 81, 766-776. [CrossRef]

38. Mediwaththe, C.; Stephens, E.; Smith, D.; Mahanti, A. Competitive energy trading framework for demand-side management in neighborhood area networks. IEEE Trans. Smart Grid 2017. [CrossRef] 
39. Jalali, M.M.; Kazemi, A. Evaluation of demand-side management over pricing competition of multiple suppliers having heterogeneous energy sources. Energies 2017, 10, 1342-1358.

40. Yu, M.; Hong, S.H. Incentive-based demand response considering hierarchical electricity market: A Stackelberg game approach. Appl. Energy 2017, 203, 267-279. [CrossRef]

41. Schrijver, A. Theory of Linear and Integer Programming; John Wiley Sons: New York, NY, USA, 1998.

42. Boyd, S.; Xiao, L.; Mutapcic, A. Subgradient Methods; notes for EE392e; Standford University, Stanford, CA, USA, 2003.

(c) 2018 by the authors. Licensee MDPI, Basel, Switzerland. This article is an open access article distributed under the terms and conditions of the Creative Commons Attribution (CC BY) license (http:/ / creativecommons.org/licenses/by/4.0/). 